\title{
Adverse Events associated with the use of cervical spine manipulation or mobilization and patientcharacteristics: a Systematic Review
}

\section{Kranenburg, H.A., ${ }^{a, b, c}$ Schmitt, M.A.a,d, Luijckx G.J.' ${ }^{\text {, Puentedura E.J.e, Van der Schans, C.P. }}$.}

${ }^{a}$ Research Group Healthy Ageing, Allied Health Care and Nursing, Hanze University of Applied Sciences, Groningen, the Netherlands

${ }^{b}$ University of Groningen University Medical Center Groningen, department of Neurology, Groningen, the Netherlands

c University of Groningen, University Medical Center Groningen, Department of Rehabilitation, Groningen, the Netherlands

' Rotterdam University of Applied Science, Research Center Innovations in Care, Rotterdam, The Netherlands

e University of Nevada Las Vegas, School of Allied Health Sciences, Department of Physical Therapy, Las Vegas, USA

Background: Cervical spinal manipulation (CSM) and cervical mobilization are frequently used in patients with neck pain and headache. Pre-manipulative cervical instability and arterial integrity tests appear to be unreliable in identifying patients at risk at risk for adverse events. It would be valuable if patients at risk could be identified by specific characteristics during the preliminary screening.

Aims: To identify 1) major adverse events (MAE) occurring after CSM or cervical mobilization and characteristics of 2) patients, 3) practitioners, 4) treatment process.

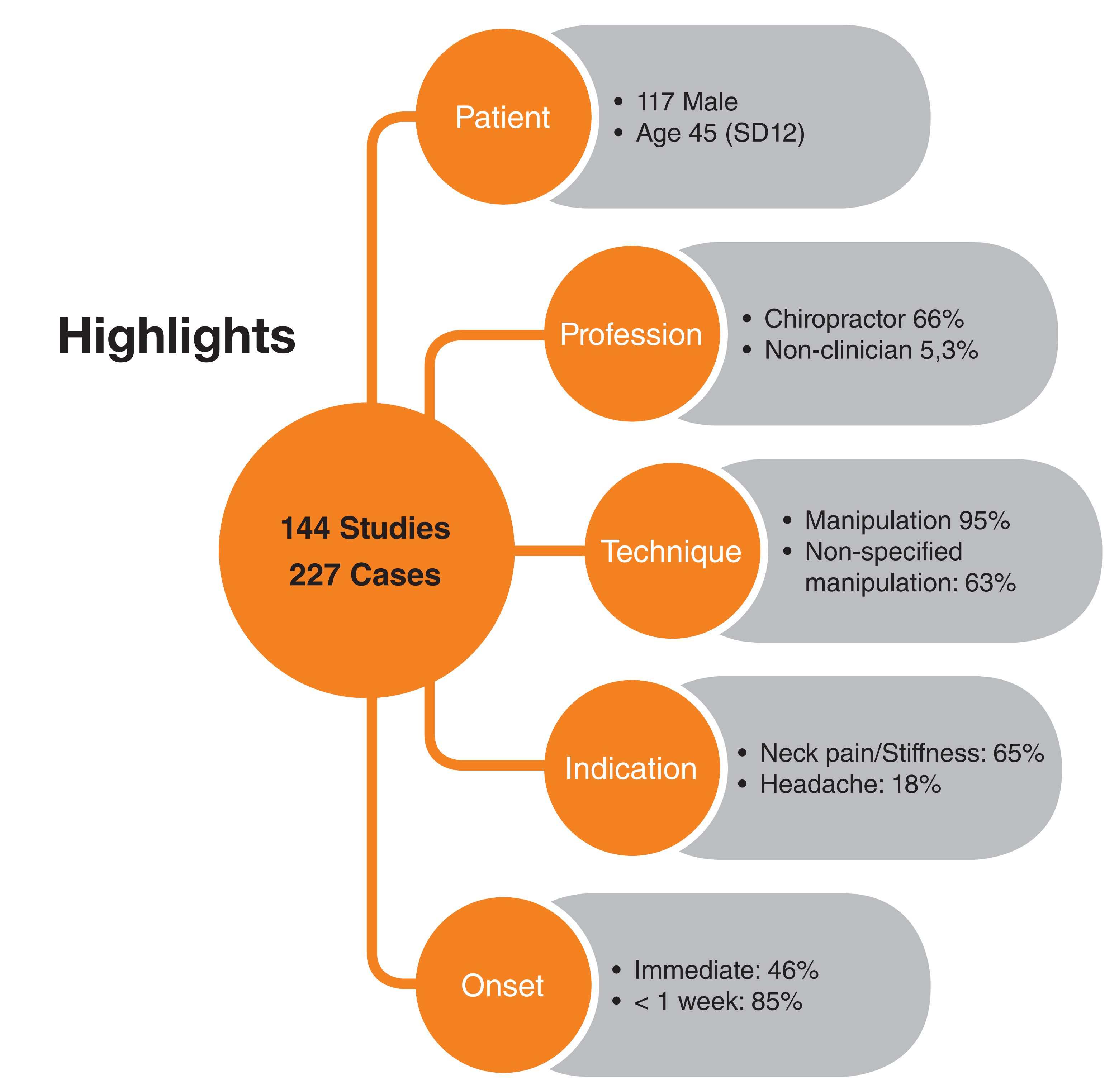

Contact details:

h.a.kranenburg@pl.hanze.nl
Summary of review: Systematic search in PubMed, Embase, CINAHL, Web-ofscience, AMED, and ICL up to December 2014. Initial 1043 studies, 144 studies were included, containing 227 cases. Cervical arterial dissection (CAD) was reported in $57 \%(P=0.21)$ of the cases and $45.8 \%$ had immediate onset symptoms. The overall distribution of gender for CAD is $55 \%(n=71)$ for female and therefore opposite of the total MAE.

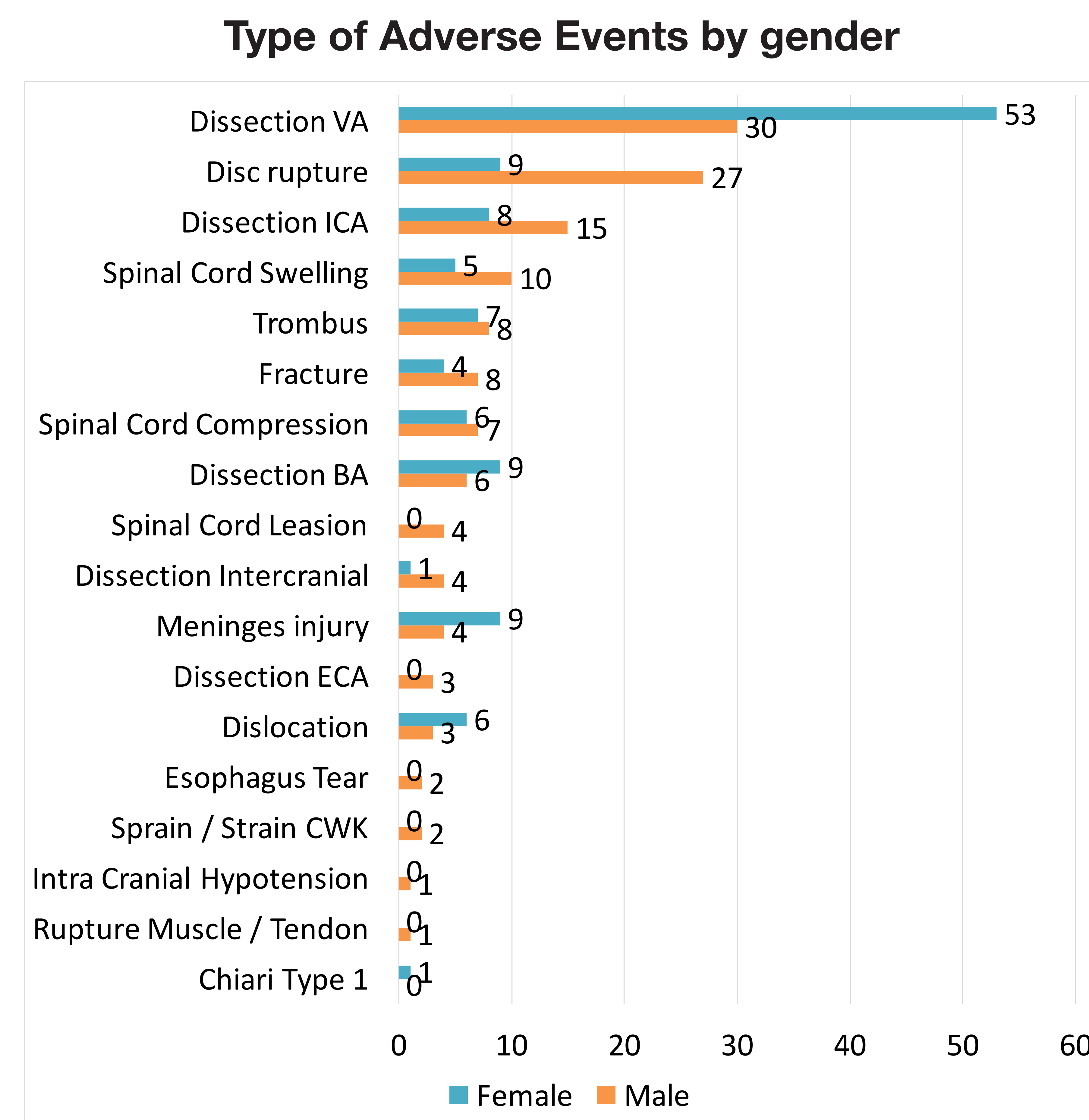

Conclusions: Patient characteristics were described poorly. No clear patient profile, related to the risk of AE after CSM, could be extracted. However, women seem more at risk for CAD. There seems to be under-reporting of cases. Further research should focus on a more uniform and complete registration of $A E$ using standardized terminology.

\section{Described parameters per case}

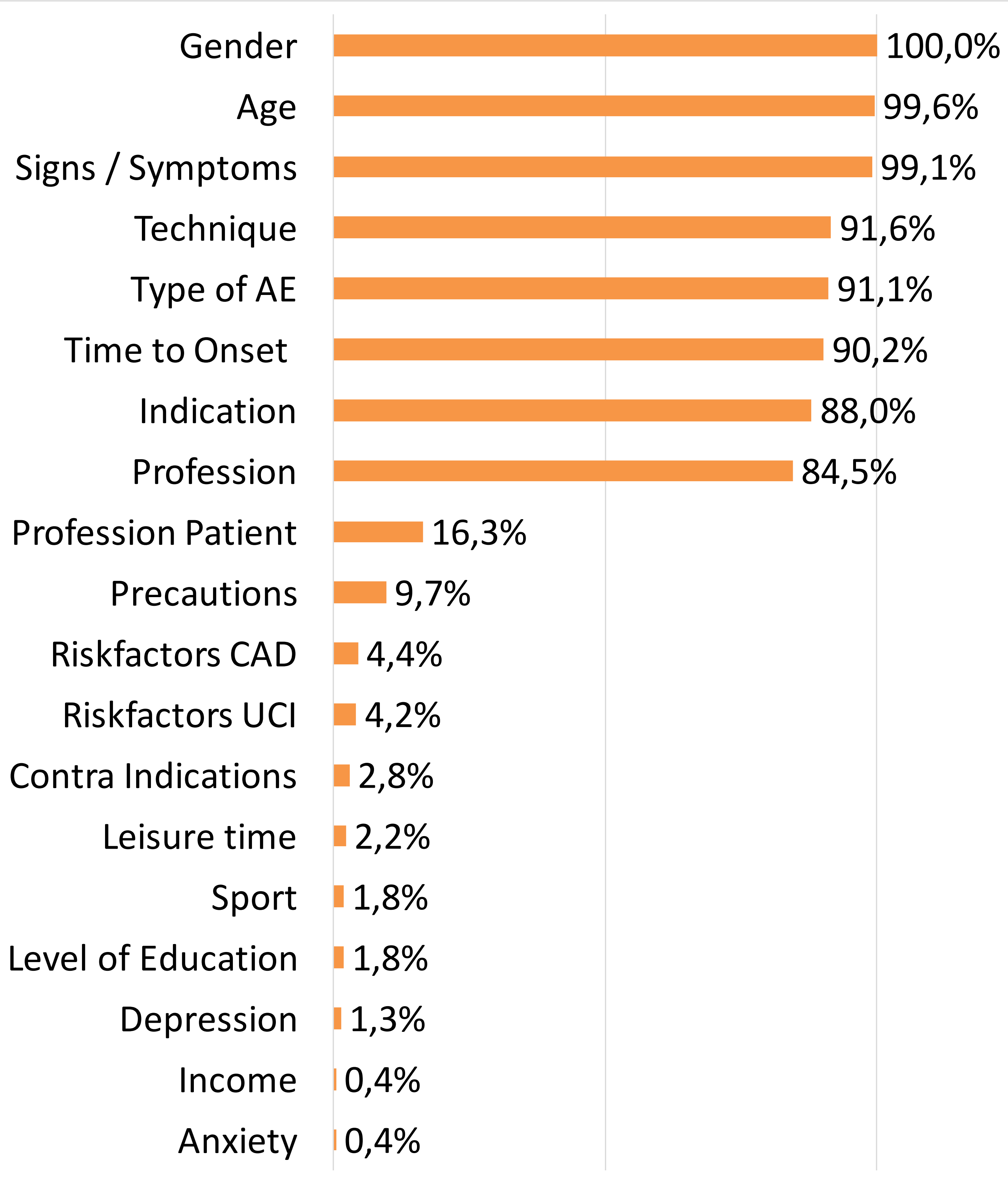

$0,0 \% \quad 50,0 \% \quad 100,0 \%$ 